\title{
An Algorithm for Multi-Parametric Quadratic Programming and Explicit MPC Solutions
}

\author{
P. Tøndel ${ }^{1}$, T.A. Johansen ${ }^{1}$, A. Bemporad ${ }^{2}$
}

\begin{abstract}
Explicit solutions to constrained linear MPC problems can be obtained by solving multi-parametric quadratic programs (mp-QP) where the parameters are the components of the state vector. We study the properties of the polyhedral partition of the state-space induced by the multiparametric piecewise linear solution and propose a new mp-QP solver. Compared to existing algorithms, our approach adopts a different exploration strategy for subdividing the parameter space, avoiding unnecessary partitioning and QP problem solving, with a significant improvement of efficiency.
\end{abstract}

\section{Introduction}

Our motivation for investigating multi-parametric quadratic programming (mp-QP) comes from linear Model Predictive Control (MPC). This refers to a class of control algorithms that compute a manipulated variable trajectory from a linear process model to minimize a quadratic performance index subject to linear constraints on a prediction horizon. The first control input is then applied to the process. At the next sample, measurements are used to update the optimization problem, and the optimization is repeated. In this way, this becomes a closed-loop approach. There has been some limitation to which processes MPC could be used on, due to the computationally expensive on-line optimization which was required. There has recently been derived explicit solutions to the constrained MPC problem, which could increase the area of use for this kind of controllers. Independent works by [1], [2], [3] and [4] has reported how a piecewise linear (PWL) solution can be computed off-line, while the on-line effort is limited to evaluate this PWL function. In particular, in [1] and [2] such a PWL function is obtained by treating the MPC optimization problem as a parametric program. Parametric programming is a term for solving an optimization problem for a range of parameter values. One can distinguish between parametric programs, in which only one parameter is considered, and multi-parametric programs, in which a vector of parameters is considered. The algorithm reported in [1] is the only mp-QP algorithm known to the authors for solving general linear MPC problems, while single parameter parametric QP is treated in [5]. Multi-parametric LP (mp-LP) is treated in [6] and [7], mp-LP in connection with MPC based on linear programming is investigated in [8], and multi-parametric mixed-integer linear programming [9] is used in [10] for obtaining explicit solutions to hybrid MPC. The problem of reducing the complexity of the

\footnotetext{
${ }^{1}$ Department of Engineering Cybernetics, Norwegian University of Science and Technology, 7491 Trondheim, Norway, Petter.Tondel@itk.ntnu.no, Tor.Arne.Johansen@itk. ntnu.no.

${ }^{2}$ Dipartimento di Ingegneria dell'Informazione, University of Siena, 53100 Siena, Italy, bemporadedii.unisi.it. ETH Zentrum, 8092 Zurich, Switzerland
}

PWL solution to linear quadratic MPC problems is addressed in $[11,12]$, and efficient on-line computation schemes of explicit MPC controllers are proposed in [13]. This paper extends the theoretical results of [1], by analyzing several properties of the geometry of the polyhedral partition and its relation to the combination of active constraints at the optimum of the quadratic program. Based on these results, we derive a new exploration strategy for subdividing the parameter space, which avoids (i) unnecessary partitioning, (ii) the solution to LP problems for determining an interior point in each new region of the parameter space, and (iii) the solution to the QP problem for such an interior point. As a consequence, there is a significant improvement of efficiency with respect to the algorithm of [1].

\section{From Linear MPC to an mp-QP Problem}

The main aspects of formulating a linear MPC problem as a multi-parametric QP will, for convenience, be repeated here. See [1] for further details. Consider the linear system

$$
\begin{aligned}
x(t+1) & =A x(t)+B u(t) \\
y(t) & =C x(t)
\end{aligned}
$$

where $x(t) \in \mathbb{R}^{n}$ is the state variable, $u(t) \in \mathbb{R}^{m}$ is the input variable, $A \in \mathbb{R}^{n \times n}, B \in \mathbb{R}^{n \times m}$ and $(A, B)$ is a controllable pair. For the current $x(t)$, MPC solves the optimization problem

$$
\begin{array}{ll}
\min _{U} & \left\{J(U, x(t))=x_{t+N \mid t}^{T} P x_{t+N \mid t}\right. \\
& \left.+\sum_{k=0}^{N-1} x_{t+k \mid t}^{T} Q x_{t+k \mid t}+u_{t+k}^{T} R u_{t+k}\right\} \\
\text { s.t. } & y_{\min } \leq y_{t+k \mid t} \leq y_{\max }, k=1, \ldots, N \\
& u_{\min } \leq u_{t+k} \leq u_{\max }, k=0, \ldots, M-1 \\
& u_{t+k}=K x_{t+k \mid t}, M \leq k \leq N-1 \\
& x_{t \mid t}=x(t) \\
& x_{t+k+1 \mid t}=A x_{t+k \mid t}+B u_{t+k}, k \geq 0 \\
& y_{t+k \mid t}=C x_{t+k \mid t}, k \geq 0
\end{array}
$$

with respect to $U \triangleq\left\{u_{t}, \ldots, u_{t+M-1}\right\}$, where $R=R^{\prime} \succ 0$, $Q=Q^{\prime} \succeq 0, P=P^{\prime} \succ 0$. When the final cost matrix $P$ and gain $K$ are calculated from the algebraic Riccati equation, under the assumption that the constraints are not active for $k \geq N$, (2) exactly solve the constrained (infinite-horizon) LQR problem for (1) with weights $Q, R$ (see also [14], [15] and [16]). This and related problems can by some algebraic manipulation be reformulated as

$$
\begin{gathered}
V_{z}(x(t))=\min _{z} \frac{1}{2} z^{T} H z \\
\text { s.t. } G z \leq W+S x(t)
\end{gathered}
$$

where $z \triangleq U+H^{-1} F^{T} x(t), U=\left[u_{t}^{T}, \ldots, u_{t+M-1}^{T}\right]^{T}$, and $x(t)$ is the current state, which can be treated as a vector of 
parameters. Note that $H \succ 0$ since $R \succ 0$. The number of inequalities is denoted by $q$ and the number of free variables is $n_{z}=m \cdot N$. Then $z \in \mathbb{R}^{n_{z}}, H \in \mathbb{R}^{n_{z} \times n_{z}}, G \in \mathbb{R}^{q \times n_{z}}$, $W \in \mathbb{R}^{q \times 1}, S \in \mathbb{R}^{q \times n}, F \in \mathbb{R}^{n \times q}$. The problem we consider here is to find the solution of the optimization problem (3)-(4) in an explicit form $z^{*}=z^{*}(x(t))$. Bemporad et. al. [1] showed that the solution $z^{*}(x(t))$ (and $U^{*}(x(t))$ ) is a continuous PWL function ${ }^{1}$ defined over a polyhedral partition of the parameter space, and $V_{z}(x(t))$ is a convex (and therefore continuous) piecewise quadratic function.

\section{Background on mp-QP}

As shown in [1], the mp-QP problem (3)-(4) can be solved by applying the Karush-Kuhn-Tucker (KKT) conditions

$$
\begin{aligned}
H z+G^{T} \lambda & =0, \lambda \in \mathbb{R}^{q}, \\
\lambda_{i}\left(G^{i} z-W^{i}-S^{i} x\right) & =0, i=1, \ldots, q, \\
\lambda & \geq 0, \\
G z-W-S x & \leq 0 .
\end{aligned}
$$

For ease of notation we write $x$ instead of $x(t)$. Superscript $i$ on some matrix denotes the $i^{\text {th }}$ row. Since $H$ has full rank, (5) gives

$$
z=-H^{-1} G^{T} \lambda
$$

Definition 1 Let $z^{*}(x)$ be the optimal solution to (3)-(4) for a given $x$. We define active constraints the constraints with $G^{i} z^{*}(x)-W^{i}-S^{i} x=0$, and inactive constraints the constraints with $G^{i} z^{*}(x)-W^{i}-S^{i} x<0$. The optimal active set $\mathcal{A}^{*}(x)$ is the set of indices of active constraints at the optimum $\mathcal{A}^{*}(x)=\left\{i \mid G^{i} z^{*}(x)=W^{i}+S^{i} x\right\}$. We also define as weakly active constraint an active constraint with an associated zero Lagrange multiplier $\lambda^{i}$, and as strongly active constraint an active constraint with a positive Lagrange multiplier $\lambda^{i}$.

Let $\breve{\lambda}$ be the Lagrange multipliers of the inactive constraints, $\breve{\lambda}=0$, and $\tilde{\lambda}$ the Lagrange multipliers of the active constraints, $\tilde{\lambda} \geq 0$. Assume for the moment that we know which constraints are active at the optimum for a given $x$. We can now form matrices $\tilde{G}, \tilde{W}$ and $\tilde{S}$ which contains the rows $G^{i}$, $W^{i}$ and $S^{i}$ corresponding to the active constraints.

Definition 2 For an active set, we say that the linear independence constraint qualification (LICQ) holds if the set of active constraint gradients are linearly independent, i.e., $\tilde{G}$ has full row rank.

Assume that LICQ holds, such that the rows of $\tilde{G}$ are linearly independent. For the active constraints, (6) and (9) give $-\tilde{G} H^{-1} \tilde{G}^{T} \tilde{\lambda}-\tilde{W}-\tilde{S} x=0$, which leads to

$$
\tilde{\lambda}=-\left(\tilde{G} H^{-1} \tilde{G}^{T}\right)^{-1}(\tilde{W}+\tilde{S} x) .
$$

Eq. (10) can now be substituted into (9) to obtain

$$
z=H^{-1} \tilde{G}^{T}\left(\tilde{G} H^{-1} \tilde{G}^{T}\right)^{-1}(\tilde{W}+\tilde{S} x) .
$$

\footnotetext{
${ }^{1}$ Strictly speaking "piecewise affine" would be a more appropriate term.
}
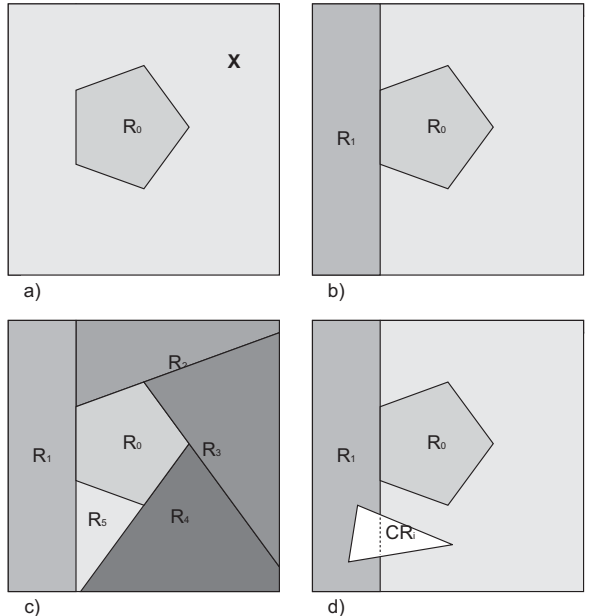

Figure 1: State-space exploration strategy.

We have now characterized the solution to (3)-(4) for a given optimal active set $A^{*} \subseteq\{1, \ldots, q\}$, and a fixed $x$. However, as long as $A^{*}$ remains the optimal active set in a neighborhood of $x$, the solution (11) remains optimal, when $z$ is viewed as a function of $x$. Such a neighborhood where $A^{*}$ is optimal is determined by imposing that $z$ must remain feasible (8)

$$
G H^{-1} \tilde{G}^{T}\left(\tilde{G} H^{-1} \tilde{G}^{T}\right)^{-1}(\tilde{W}+\tilde{S} x) \leq W+S x .,
$$

and that the Lagrange multipliers $\lambda$ must remain nonnegative (7)

$$
-\left(\tilde{G} H^{-1} \tilde{G}^{T}\right)^{-1}(\tilde{W}+\tilde{S} x) \geq 0 . .
$$

Equations (12) and (13) describe a polyhedron in the state space. This region is denoted as the critical region $C R_{0}$ corresponding to the given set $A^{*}$ of active constraints, is a convex polyhedral set, and represents the largest set of parameters $x$ such that the combination $A^{*}$ of active constraints at the minimizer is optimal [1].

The recursive algorithm of [1] can be briefly summarized as follows: Choose a parameter $x_{0}$. Solve a QP to find the optimal active set $\mathcal{A}_{0}$ for $x_{0}$, and then use (10)-(13) to characterize the solution and critical region corresponding to $\mathcal{A}_{0}$. Then divide the parameter space as in Figure 1 by reversing one by one the hyperplanes defining the critical region. Iteratively subdivide each new region $R_{i}$ in a similar way. The main drawback of this algorithm is that the regions $R_{i}$ are not related to optimality, as they can split some of the critical regions like $C R_{1}$ in Figure 1d. A consequence is that $C R_{1}$ will be detected at least twice.

The following theorem characterizes the primal and dual parametric solutions, and will be useful in the sequel.

Theorem 1 Consider Problem (3)-(4) with $H \succ 0$. Let $X \in \mathbb{R}^{n}$ be a polyhedron. Then the solution $z^{*}(x)$ and the Lagrange multipliers $\lambda^{*}(x)$ of a $m p-Q P$ are piecewise linear, functions of the parameters $x$, and $z^{*}(x)$ is continuous. Moreover, if LICQ holds for all $x \in X, \lambda^{*}(x)$ is also continuous. 
Proof: Follows easily from uniqueness (due to $H \succ 0$ and LICQ) of $z^{*}(x)$ and $\lambda^{*}(x)$, cf. [1], [17].

\section{Characterization of the Partition}

Below, we denote by $z_{k}^{*}(x)$ the linear expression of the PWL function $z^{*}(x)$ over the critical region $C R_{k}$.

Definition 3 Let a polyhedron $X \subset \mathbb{R}^{n}$ be represented by the linear inequalities $A_{0} x \leq b$. Let the $i^{\text {th }}$ hyperplane, $A_{0}^{i} x=b^{i}$ be denoted by $\mathcal{H}$. If $X \cap \mathcal{H}$ is $(n-1)$-dimensional then $X \cap \mathcal{H}$ is called a facet of the polyhedron.

Definition 4 Two polyhedra are called neighboring polyhedra if they have a common facet.

Definition 5 Let a polyhedron $X$ be represented by $A_{0} x \leq$ b. We say that $A_{0}^{i} x \leq b^{i}$ is redundant if $A_{0}^{j} x \leq b^{j} \forall j \neq i \Rightarrow$ $A_{0}^{i} x \leq b^{i}$ (i.e., it can be removed from the description of the polyhedron). The inequality $i$ is redundant with degree $h$ if it is redundant and there exists a h-dimensional subset $Y$ of $X$ such that $A_{0}^{i} x=b^{i}$ for all $x \in Y$.

Definition 6 A representation of a polyhedron (12)-(13) is $l$-minimal if all redundant constraints have degree $h \geq l$.

Clearly, a representation of a polyhedron $X \subset \mathbb{R}^{n}$ is $n$ minimal if it contains all inequalities defining facets, and does not contain two or more coincident hyperplanes.

Let us consider a hyperplane defining the common facet between two polyhedra $C R_{0}, C R_{i}$ in the optimal partition of the state space. There are two different kinds of hyperplanes. The first (Type I) are those described by (12), which represents a non-active constraint of (4) that becomes active at the optimum as $x$ moves from $C R_{0}$ to $C R_{i}$. As proved in the following theorem, this means that if a polyhedron is bounded by a hyperplane which originates from (12), the corresponding constraint will be activated on the other side of the facet defined by this hyperplane. In addition, the corresponding Lagrange multiplier may become positive. The other kind (Type II) of hyperplanes which bounds the polyhedra are those described by (13). In this case, the corresponding constraint will be non-active on the other side of the facet defined by this hyperplane.

Theorem 2 Consider an optimal active set $\left\{i_{1}, i_{2}, \ldots, i_{k}\right\}$ and its corresponding n-minimal representation of the critical region $C R_{0}$ obtained by (12)-(13) after removing redundant inequalities. Let $C R_{i}$ be a full-dimensional neighboring critical region to $C R_{0}$ and assume LICQ holds on their common facet $\mathcal{F}=C R_{0} \cap \mathcal{H}$ where $\mathcal{H}$ is the separating hyperplane between $C R_{0}$ and $C R_{i}$. Moreover, assume that there are no constraints which are weakly active at the optimizer $z^{*}(x)$ for all $x \in C R_{0}$. Then,

Type I. If $\mathcal{H}$ is given by $G^{i_{k+1}} z_{0}^{*}(x)=W^{i_{k+1}}+S^{i_{k+1}} x$, then the optimal active set in $C R_{i}$ is $\left\{i_{1}, \ldots, i_{k}, i_{k+1}\right\}$.

Type II. If $\mathcal{H}$ is given by $\lambda_{0}^{i_{k}}(x)=0$, then the optimal active set in $C R_{i}$ is $\left\{i_{1}, \ldots, i_{k-1}\right\}$.
Proof: Let us first prove Type I. In order for some constraint $i_{j} \in\left\{i_{1}, \ldots, i_{k}\right\}$ not to be in the optimal active set in $C R_{i}$, by continuity of $\lambda^{*}(x)$ (due to Theorem 1 and LICQ), it follows that $\left(\lambda^{*}\right)^{i_{j}}(x)=\lambda_{0}^{i_{j}}(x)=0$ for all $x \in \mathcal{F}$. Since there are no constraints which are weakly active for all $x \in C R_{0}$, this would mean that constraint $i_{j}$ becomes non-active at $\mathcal{F}$. But this contradicts the assumption of $n$-minimality since $\lambda_{0}^{i_{j}}(x) \geq 0$ and $G^{i_{k+1}} z_{0}^{*}(x) \leq W^{i_{k+1}}+S^{i_{k+1}} x$ would be coincident. On the other hand $\left\{i_{1}, \ldots, i_{k}\right\}$ can not be the optimal active set on $C R_{i}$ because $C R_{0}$ is the largest set of $x$ 's such that $\left\{i_{1}, \ldots, i_{k}\right\}$ is the optimal active set. Then, the optimal active set in $C R_{i}$ is a superset of $\left\{i_{1}, \ldots, i_{k}\right\}$. Now assume that another constraint $i_{k+2}$ is active in $C R_{i}$. That means $G^{i_{k+2}} z_{i}^{*}(x)=W^{i_{k+2}}+S^{i_{k+2}} x$ in $C R_{i}$, and by continuity of $z^{*}(x)$, the equality also holds for $x \in \mathcal{F}$. However, $G^{i_{k+2}} z_{0}^{*}(x)=W^{i_{k+2}}+S^{i_{k+2}} x$ would then coincide with $G^{i_{k+1}} z_{0}^{*}(x)=W^{i_{k+1}}+S^{i_{k+1}} x$, which contradicts the assumption of $n$-minimality. Therefore, only $\left\{i_{1}, \ldots, i_{k}, i_{k+1}\right\}$ can be the optimal active set in $C R_{i}$. The proof for Type II is similar.

Corollary 1 Consider the same assumptions as in Theorem 2 , except that the assumption of n-minimality is relaxed into $(n-1)$-minimality, i.e., two or more hyperplanes can coincide. Let $\mathcal{I} \subset\left\{i_{1}, \ldots, i_{k}\right\}$ be the set of indices corresponding to coincident hyperplanes in the $(n-1)$-minimal representation of (12)-(13) of $C R_{0}$.

- Every constraint $i_{j}$ where $i_{j} \in\left\{i_{1}, i_{2}, \ldots, i_{k}\right\} \backslash \mathcal{I}$ is active in $C R_{i}$.

- Every constraint $i_{j}$ where $i_{j} \notin\left\{i_{1}, i_{2}, \ldots, i_{k}\right\} \cup \mathcal{I}$ is inactive in $C R_{i}$.

This means that every combination of the indices in $\mathcal{I}$ cannot be excluded directly. We remark that coincident hyperplanes are rare, as from (12)-(13) one can see that special structures of $H, F, G, W$, and $S$ are required for two or more hyperplanes to be coincident. Anyway, when for instance two hyperplanes are coincident, by Corollary 1 there are three possible active sets which have to be checked to find the optimal active set in $C R_{i}$.

Example 1. Consider the double integrator [3]

$$
A=\left[\begin{array}{cc}
1 & T_{s} \\
0 & 1
\end{array}\right], \quad B=\left[\begin{array}{c}
T_{s}^{2} \\
T_{s}
\end{array}\right]
$$

where the sampling interval $T_{s}=0.05$, and consider the MPC problem over the prediction horizon $N=2$ with cost matrices $Q=\left[\begin{array}{ll}1 & 0 \\ 0 & 0\end{array}\right], R=1$. The constraints in the system are $-0.5 \leq x_{2} \leq 0.5,-1 \leq u \leq 1$. The mp-QP associated with this problem has the form (3)-(4) with

$$
\begin{gathered}
H=\left[\begin{array}{lll}
1.079 & 0.076 \\
0.076 & 1.073
\end{array}\right], F=\left[\begin{array}{ccccc}
1.109 & 1.036 \\
1.573 & 1.517
\end{array}\right], \\
G^{T}=\left[\begin{array}{ccccccccc}
1 & 0 & -1 & 0 & 0.05 & 0.05 & -0.05 & -0.05 \\
0 & 1 & 0 & -1 & 0 & 0.05 & 0 & -0.05
\end{array}\right], \\
W^{T}=\left[\begin{array}{llllllll}
1 & 1 & 1 & 1 & 0.5 & 0.5 & 0.5 & 0.5
\end{array}\right], S^{T}= \\
{\left[\begin{array}{cccccccc}
1.0 & 0.9 & -1.0 & -0.9 & 0.1 & 0.1 & -0.1 & -0.1 \\
1.4 & 1.3 & -1.4 & -1.3 & -0.9 & -0.9 & 0.9 & 0.9
\end{array}\right]}
\end{gathered}
$$


We start the partitioning by finding the region where no con-
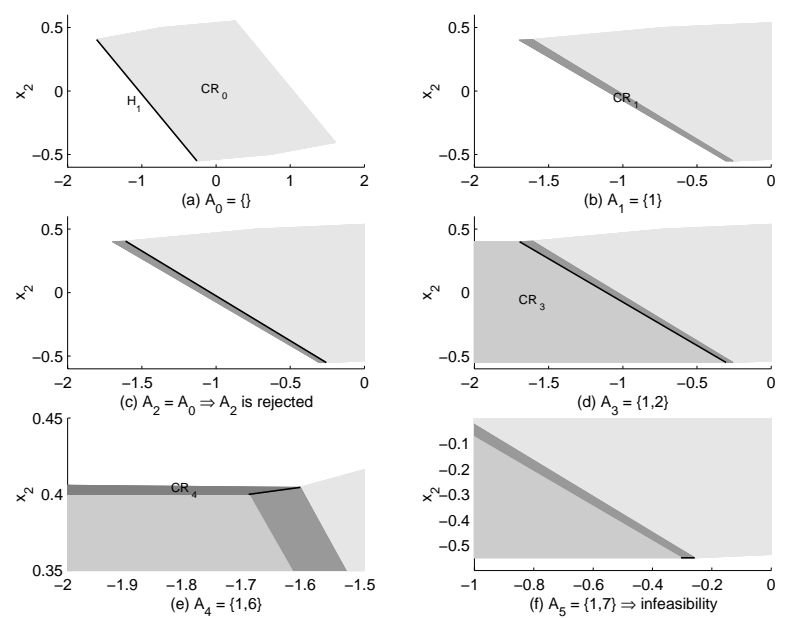

Figure 2: Critical regions for double integrator.

straints are active. As the mp-QP is created from a feasible MPC problem, the empty active set will be optimal in some full-dimensional region $\left(\mathcal{A}_{0}=\emptyset\right.$, and $\tilde{G}, \tilde{W}$ and $\tilde{S}$ are empty matrices, $z^{*}(x)=0$ and the first component of $U^{*}(x)$ is the unconstrained LQR gain). This critical region is then described by $0 \leq W+S x$, which contains eight inequalities. Two of these inequalities are redundant with degree 0 (\#2 and \#4), the remaining six hyperplanes are facet inequalities of the polyhedron (see Figure $2 \mathrm{a}$ ). By crossing the facet given by $\mathcal{H}_{1}$, defined by inequality 1 and of Type I, as predicted by Theorem 2 the optimal active set across this facet is $\mathcal{A}_{1}=\{1\}$, which leads to the critical region $C R_{1}$ (see Figure $2 b)$. After removing redundant inequalities we are left with a $n$-minimal representation of $C R_{1}$ containing four facets. The first of these is of Type II, $\lambda^{1}(x)=0$. The other three are of Type I. These are inequalities \#2, \#6 and \#7. Consider first the other side of the facet which comes from $\lambda^{1}(x)=0$, see Figure 2c. The region should not have constraint $1 \mathrm{ac}-$ tive, so the optimal active set is $\mathcal{A}_{2}=\emptyset$. This is the same combination of active constraints as $\mathcal{A}_{0}$, as expected, so $\mathcal{A}_{2}$ is not pursued. Next, consider crossing the respective facets of inequalities 2, 6 and 7, see Figures $2 \mathrm{~d}-2 \mathrm{f}$. This results in three different active sets: $\mathcal{A}_{3}=\{1,2\}, \mathcal{A}_{4}=\{1,6\}$ and $\mathcal{A}_{5}=\{1,7\} . \mathcal{A}_{3}$ and $\mathcal{A}_{4}$ leads to new polyhedra as shown in the figures. The combination $\mathcal{A}_{5}$ leads to an interesting case of "degeneracy". The associated matrix $\tilde{G}$ has linearly dependent rows, which violates the LICQ assumption. In this case, $\mathcal{A}_{5}$ leads to an infeasible part of the state space. A general treatment of degeneracy is given in the next section.

Theorem 2 and Corollary 1 show how to find the optimal active set across a facet only by using the knowledge of which kind of hyperplane the facet corresponds to, except in degenerate cases, which is the topic of the next section.

\section{Degeneracy in mp-QP}

We have so far assumed that LICQ holds on the common facet between two polyhedra, and that there are no constraints which are weakly active for all $x$ within a critical region.
Such cases are referred to as degenerate. We will first consider how to handle cases where LICQ is violated, and then consider weakly active constraints.

Theorem 3 Consider a generic combination $A^{*} \subseteq$ $\{1, \ldots, q\}$ of active constraints and assume that the corresponding rows $[\tilde{G}-\tilde{S}-\tilde{W}]$ are linearly independent. If $L I C Q$ is violated, then the corresponding critical region is not full-dimensional.

Proof: Let the active constraints be $\tilde{G} z=\tilde{S} x+\tilde{W}$. Since LICQ is violated, $\tilde{G}$ has not full rank and a reduced set of equations can be defined without changing the solution $z^{*}(x): G^{\prime} z=S^{\prime} x+W^{\prime}$. Assume without loss of generality that

$$
\tilde{G}=\left[\begin{array}{c}
G^{\prime} \\
G^{k}
\end{array}\right], \tilde{S}=\left[\begin{array}{c}
S^{\prime} \\
S^{k}
\end{array}\right], \tilde{W}=\left[\begin{array}{c}
W^{\prime} \\
W^{k}
\end{array}\right],
$$

where $G^{k}$, and $S^{k}$ are row-vectors and $W^{k}$ is a scalar. Let $C R^{\prime}$ and $\widetilde{C R}$ be the critical regions where the active sets corresponding to $G^{\prime}$ and $\tilde{G}$, respectively, are optimal. The solution is $z_{C R^{\prime}}^{*}(x)=z_{\overparen{C R}}^{*}(x)=L x+v$ within both $C R^{\prime}$ and $\widetilde{C R}$, where $L=H^{-1} G^{\prime T}\left(G^{\prime} H^{-1} G^{\prime T}\right)^{-1} S^{\prime}$, $v=H^{-1} G^{\prime T}\left(G^{\prime} H^{-1} G^{\prime T}\right)^{-1} W^{\prime}$. It is clear that $\widetilde{C R} \subset$ $\left\{x \in \mathbb{R}^{n} \mid[\tilde{G}-\tilde{S}]\left[\begin{array}{c}z_{\widetilde{C R}}^{*}(x) \\ x\end{array}\right]=\tilde{W}\right\}=\tilde{M}$ and

$$
\begin{aligned}
\tilde{M} & =\left\{x \in \mathbb{R}^{n} \mid\left[\begin{array}{cc}
G^{\prime} & -S^{\prime} \\
G^{k} & -S^{k}
\end{array}\right]\left[\begin{array}{c}
L x+v \\
x
\end{array}\right]=\left[\begin{array}{c}
W^{\prime} \\
W^{k}
\end{array}\right]\right\} \\
& =\left\{\begin{array}{c}
x \in \mathbb{R}^{n} \mid G^{\prime} L x-S^{\prime} x+G^{\prime} v=W^{\prime} \\
,\left(G^{k} L-S^{k}\right) x+G^{k} v=W^{k}
\end{array}\right\} \\
& =\left\{x \in \mathbb{R}^{n} \mid\left(G^{k} L-S^{k}\right) x+G^{k} v=W^{k}\right\} .
\end{aligned}
$$

If $G^{k} L \neq S^{k}$ or $G^{k} v \neq W^{k}$ it follows that $\tilde{M}$ is not a full-dimensional subspace of $\mathbb{R}^{n}$, and since $\widetilde{C R} \subset$ $\tilde{M}$, neither is $\widetilde{C R}$. Suppose this does not hold, i.e., $G^{k} L=S^{k}$ and $G^{k} v=W^{k}$. Since $\tilde{G}$ has not full rank, $G^{k}=\alpha G^{\prime}$, where $\alpha$ is a row-vector, and $S^{k}=\alpha G^{\prime} H^{-1} G^{\prime T}\left(G^{\prime} H^{-1} G^{\prime T}\right)^{-1} S^{\prime}=\alpha S^{\prime}, W^{k}=$ $\alpha G^{\prime} H^{-1} G^{\prime T}\left(G^{\prime} H^{-1} G^{\prime T}\right)^{-1} W^{\prime}=\alpha W^{\prime}$. Then, there is linear dependence between rows of $\left[\begin{array}{lll}\tilde{G} & -\tilde{S} & -\tilde{W}\end{array}\right]$, a contradiction.

In an MPC problem one might avoid full-dimensional critical regions with violation of LICQ by simply slightly perturbing the weight matrices and the constraints, without producing significant changes of the closed-loop behavior. On the other hand, in some situations this may not be possible, for instance equality constraints such as terminal state constraints $x_{t+N \mid t}=0$, would lead to violation of LICQ (cf. [5, Example 6.3]). In such cases, full-dimensional critical regions can be handled by solving a QP, as in [18]. One should also a priori remove redundant constraints from $[G-S]\left[\begin{array}{c}z \\ x\end{array}\right] \leq W$, which might remove linear dependence in $[\tilde{G}-\tilde{S}-\tilde{W}]$, and also would reduce the complexity of the mp-QP.

Next Theorem 4 provides a method to find the optimal active set in a neighboring region also when LICQ is violated on the 
common facet. Before proceeding further, we need a technical Lemma, which is proven in [18]. Since now on, when we discuss matrices as $\tilde{G}, \tilde{S}$, or $\tilde{W}$ or the active constraints $\tilde{\lambda}$, according to different active sets, we will use the notation $\tilde{G}^{\left\{i_{1}, \ldots, i_{k}\right\}}$ to mean the rows of the $G$ matrix corresponding to the active set $\left\{i_{1}, \ldots, i_{k}\right\}$, etc.

Lemma 1 Let the optimal active set in a critical region $C R_{0}$ be $\left\{i_{1}, \ldots, i_{k}\right\}$, and consider an n-minimal representation of $C R_{0}$. Assume that there are no constraints which are weakly active for all $x \in C R_{0}$ and that $\tilde{G}^{\left\{i_{1}, \ldots, i_{k}\right\}}$ does not have linearly dependent rows. Let $C R_{i}$ be a full-dimensional neighboring critical region to $C R_{0}$, and let $\mathcal{F}$ be their common facet with $\mathcal{F}=C R_{0} \cap \mathcal{H}$ and $\mathcal{H}$ is the Type I hyperplane $G^{i_{k+1}} z_{0}^{*}(x)=W^{i_{k+1}}+S^{i_{k+1}} x$. Suppose $\tilde{G}^{\left\{i_{1}, \ldots, i_{k}, i_{k+1}\right\}}$ has linearly dependent rows, such that LICQ is violated at $\mathcal{F}$. Then, if there is a feasible solution in $C R_{i}$, the optimal active set in $C R_{i}$ consists of constraint $i_{k+1}$ and some subset of $\left\{i_{1}, \ldots, i_{k}\right\}$.

Theorem 4 Make the same assumptions as in Lemma 1. Consider the following $L P$ :

$$
\begin{aligned}
\max \lambda^{i_{k+1}} & \\
\text { s.t. } H z+\left(\tilde{G}^{\left\{i_{1}, \ldots, i_{k}, i_{k+1}\right\}}\right)^{T} \lambda^{\left\{i_{1}, \ldots, i_{k}, i_{k+1}\right\}} & =0 \\
\lambda^{\left\{i_{1}, \ldots, i_{k}, i_{k+1}\right\}} & \geq 0
\end{aligned}
$$

If this LP has a bounded solution, the optimal active set in $C R_{i}$ consists of the elements of $\left\{i_{1}, \ldots, i_{k}, i_{k+1}\right\}$ with $\lambda^{i_{j}}>$ 0 in the solution. If the $L P$ is unbounded, $C R_{i}$ is an infeasible area of the parameter space.

Proof: The solution $z^{*}(x)$ to (5)-(8) on $\mathcal{F}$ is known from the solution in $C R_{0}$. The optimal Lagrange multipliers $\lambda^{*}(x)$ on $\mathcal{F}$ is then characterized by (17)-(18). The solution to (5)(8) in $C R_{i}$ must also be valid on $\mathcal{F}$, in particular, $\lambda_{i}^{*}(x)$ must satisfy (17)-(18) on $\mathcal{F}$. From Lemma 1, the optimal active set in $C R_{i}$, consists of constraint $i_{k+1}$ and a proper subset of $\left\{i_{1}, \ldots, i_{k}\right\}$. Therefore, there must be a solution on $\mathcal{F}$ which satisfies $\left(\lambda_{i}^{i_{k+1}}\right)^{*}(x)>0$ and $\left(\lambda_{i}^{i_{j}}\right)^{*}(x)=0$ for at least one $i_{j} \in\left\{i_{1}, \ldots, i_{k}\right\}$. With a fixed $\lambda^{i_{k+1}}=0$, (17) defines $n_{z}$ equations in $k$ unknowns $\left(n_{z} \geq k\right)$. But there exists a solution from $C R_{0}$, such that a reduced set of equations can be defined with $k$ equations in $k$ unknowns. When $\lambda^{i_{k+1}} \geq 0$, (17) consists of $k$ equations in $k+1$ unknowns, and $\lambda^{\overline{i_{j}}}=$ $f^{i_{j}}\left(\lambda^{i_{k+1}}\right)$ for any $i_{j} \in\left\{i_{1}, \ldots, i_{k}\right\}$, where $f^{i_{j}}$ is an affine function. When $\lambda^{i_{k+1}}=0$, the solution of (17)-(18) has $\lambda^{i_{j}}>0$ for all $i_{j} \in\left\{i_{1}, \ldots, i_{k}\right\}$ (due to n-minimality and no weakly active constraints for all $x$ in $C R_{0}$ ). To find a solution which satisfies Lemma $1, \lambda^{i_{k+1}}$ must be increased from zero until $\lambda^{i_{j}}=0$ for some $i_{j} \in\left\{i_{1}, \ldots, i_{k}\right\}$. This is the only solution of (17)-(18) which satisfies Lemma 1 because if $\lambda^{i_{k+1}}$ is increased further, $\lambda^{i_{j}}=f^{i_{j}}\left(\lambda^{i_{k+1}}\right)<0$ (since $f^{i_{j}}$ is an affine function).

Constraints that are weakly active for all $x$ in a critical region, can be handled according to the following result, which can be proven similarly to Theorem 2 .
Theorem 5 Make the same assumptions as in Theorem 2, except that now constraint $i_{1}$ is weakly active for all $x \in$ $C R_{0}$.

Type I. If $\mathcal{H}$ is given by $G^{i_{k+1}} z_{0}^{*}(x)=W^{i_{k+1}}+S^{i_{k+1}} x$, then the optimal active set in $C R_{i}$ is $\left\{i_{1}, \ldots, i_{k}, i_{k+1}\right\}$ or $\left\{i_{2}, \ldots, i_{k}, i_{k+1}\right\}$.

Type II. If $\mathcal{H}$ is given by $\lambda_{0}^{i_{k}}(x)=0$, then the optimal active set in $C R_{i}$ is $\left\{i_{1}, \ldots, i_{k-1}\right\}$ or $\left\{i_{2}, \ldots, i_{k-1}\right\}$.

Example 1 (cont'd). We want to show how to handle the case when LICQ is violated at a facet. First, notice in Figure 2 that the polyhedra made from $\mathcal{A}_{3}$ and $\mathcal{A}_{4}$ are neighboring polyhedra, but still there are two elements in $\mathcal{A}_{3}$ which are different from $\mathcal{A}_{4}$. This is caused by a violation of LICQ on the hyperplane separating these regions. Assume we have found $C R_{3}$, and try to detect $C R_{4}$. We cross a hyperplane of Type 1 , which defines their common facet $\mathcal{F}$. This hyperplane says that constraint 6 is becoming active at the optimal solution for $x \in \mathcal{F}$. Since constraint 1 and 2 was active in $C R_{3}$, constraints $\{1,2,6\}$ are active at the optimal solution for $x \in \mathcal{F}$. This obviously leads to linear dependence among the elements in $\tilde{G}$, and Theorem 4 is applied to find the optimal active set across $\mathcal{F}$. A point $x_{0} \in \mathcal{F}$ is needed to initialize the LP (16)-(18), and in this case we use $x_{0}=\left[\begin{array}{ll}-1.8 & 0.4\end{array}\right]^{T}$. We then solve the LP

$$
\begin{array}{r}
\max \lambda^{6} \\
\text { s.t.Hz+(} \left.\tilde{G}^{\{1,2,6\}}\right)^{T} \lambda^{\{1,2,6\}}=0, \lambda^{\{1,2,6\}}>0
\end{array}
$$

From (11), $H z=\left(\tilde{G}^{\{1,2\}}\right)^{T}\left(\tilde{G}^{\{1,2\}} H^{-1}\left(\tilde{G}^{\{1,2\}}\right)^{T}\right)^{-1}$. $\left(\tilde{W}^{\{1,2\}}+\tilde{S}^{\{1,2\}} x_{0}\right)$, and the solution of (19) is $\lambda^{\{1,2,6\}}=$ $\left[\begin{array}{lll}0.11 & 0 & 4.25\end{array}\right]^{T}$. Hence, $\lambda^{2}$ should be removed from the active set, and the optimal active set in $C R_{4}$ is $\{1,6\}$, as expected. Next, consider crossing the facet drawn as a thick segment in Figure 2f. The optimal active set in $C R_{1}$ is $\{1\}$, and the inequality corresponding to the facet says that constraint 7 is being activated. $G^{1}$ and $G^{7}$ are linearly dependent, so LICQ is violated. We therefore solve the LP (16)(18), with $x_{0}=\left[\begin{array}{ll}-0.28 & -0.55\end{array}\right]^{T}: \max \lambda^{7}$, s.t. $H z+$ $\left(\tilde{G}^{\{1,7\}}\right)^{T} \lambda^{\{1,7\}}=0, \quad \lambda^{\{1,7\}}>0$. The solution to this LP is unbounded and according to Theorem 4 , we have reached an infeasible part of the state space, which is easily verified.

\section{Off-Line Mp-QP Algorithm}

Based on the results of Sections 3, 4 and 5, we finally present an efficient algorithm for the computation of the solution to the mp-QP (3)-(4). Generally, there exist active sets which are not optimal anywhere in the parameter space (typically, most active sets are not optimal anywhere). We need an active set which is optimal in a full-dimensional region to start the algorithm below. Generally we can do this by choosing a feasible $x$, and find the optimal active set for this $x$ by solving a QP. A special case is when we solve a linear MPC 
Table 1: Computation times, double integrator example.

\begin{tabular}{|c|c|c|c|}
\hline \hline Hor. & Alg. from [1] & Alg. 1 & \# regions \\
\hline \hline 2 & $0.77 \mathrm{~s}$ & $0.13 \mathrm{~s}$ & 9 \\
\hline 3 & $2.63 \mathrm{~s}$ & $0.22 \mathrm{~s}$ & 19 \\
\hline 4 & $5.60 \mathrm{~s}$ & $0.38 \mathrm{~s}$ & 33 \\
\hline 5 & $9.01 \mathrm{~s}$ & $0.65 \mathrm{~s}$ & 51 \\
\hline 6 & $16.48 \mathrm{~s}$ & $1.06 \mathrm{~s}$ & 73 \\
\hline
\end{tabular}

problem. Then there will be a region where the unconstrained controller will be optimal, and we can choose the active set $\mathcal{A}_{0}=\emptyset$ (see [8], Prop. 2).

Let $L_{\text {cand }}$ be a list of active sets which are found, but not yet explored (i.e., are candidates for optimality) and $L_{\text {opt }}$ be the set of active sets which have been explored (i.e., are found to be optimal).

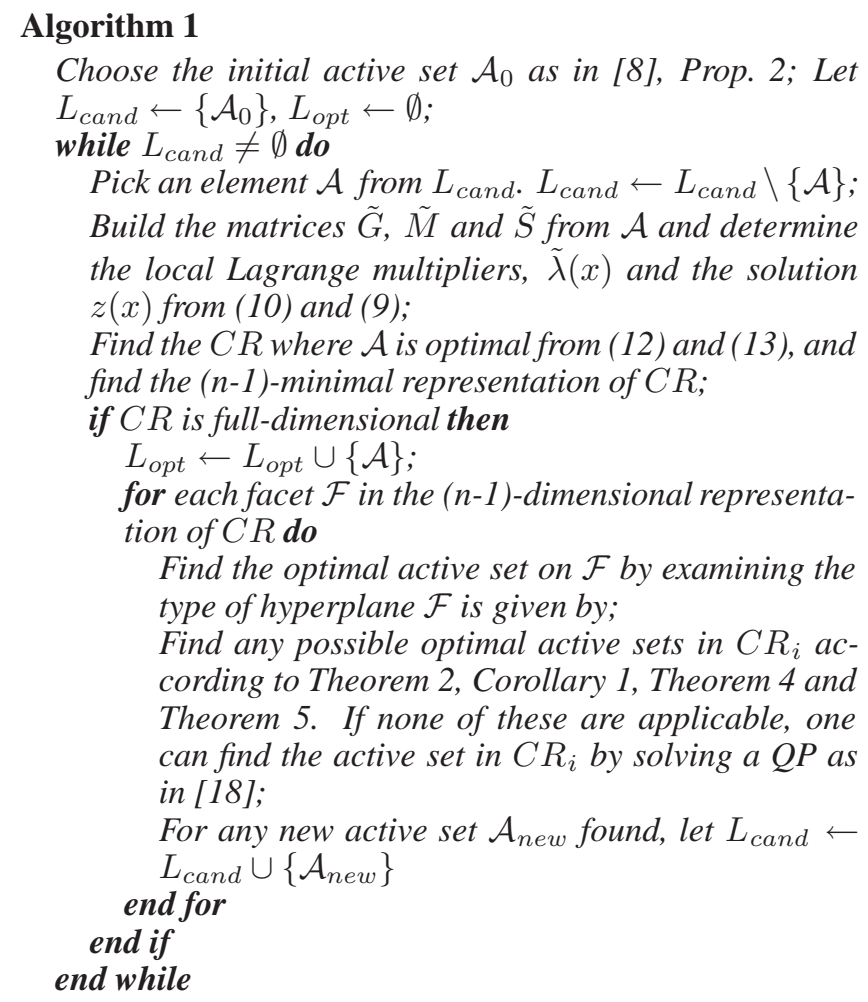

Example 2. For comparing the efficiency of Algorithm 1 and the algorithm of [1] we have repeated the double integrator example from [1]. Some corrections for different CPU frequency are made. ${ }^{2}$ Symmetries in the MPC problem are here exploited, almost halving the computation times.In this example more than $60 \%$ of the time is spent by both algorithms on removing redundant constraints from the polyhedra. This is done by solving one LP for each hyperplane in each polyhedron.

\section{Conclusions}

In this paper we have proposed a new approach for solving $\mathrm{mp}-\mathrm{QP}$ problems giving off-line piecewise linear explicit so-

\footnotetext{
${ }^{2}$ Both methods were implemented in MATLAB using the NAG Foundation Toolbox to solve LP/QP subproblems.
}

lutions to MPC control problems. Being based on the exploitation of direct relations between neighboring polyhedral regions and combinations of active constraints, we believe that our contribution significantly advances the field of explicit MPC control, both theoretically and practically, as examples have indicated large improvements over existing mpQP algorithms.

\section{References}

[1] A. Bemporad, M. Morari, V. Dua, and E. N. Pistikopoulos, "The explicit linear quadratic regulator for constrained systems," Automatica, to appear.

[2] A. Bemporad, M. Morari, V. Dua, and E. Pistikopoulos, "The explicit linear quadratic regulator for constrained systems," in Proc. American Contr. Conf., (Chicago, IL), pp. 872-876, June 2000.

[3] T. A. Johansen, I. Petersen, and O. Slupphaug, "Explicit subopitmal linear quadratic regulation with input and state constraints," in Proc. 39th IEEE Conf. on Decision and Control, (Sydney), 2000. [4] M. M. Seron, J. A. D. Doná, and G. C. Goodwin, "Global analytical model predictive control with input constraints," in Proc. 39th IEEE Conf. on Decision and Control, (Sydney), 2000.

[5] A. Berkelaar, K. Roos, and T. Terlaky, "Advances in sensitivity analysis and parametric programming," pp. [6-1]-[6-45], 1997.

[6] T. Gal, Postoptimal Analyses, Parametric Programming, and Related Topics. Berlin: de Gruyter, second ed., 1995.

[7] F. Borrelli, A. Bemporad, and M. Morari, "A geometric algorithm for multi-parametric linear programming," Tech. Rep. AUTOO04, Automatic Control Laboratory, ETH, 2000.

[8] A. Bemporad, F. Borrelli, and M. Morari, "Model predictive control based on linear programming - the explicit solution," Tech. Report, ETH Zurich, AUT01-06, 2001.

[9] V. Dua and E. Pistikopoulos, "An algorithm for the solution of multiparametric mixed integer linear programming problems," Annals of Operations Research, to appear.

[10] A. Bemporad, F. Borrelli, and M. Morari, "Piecewise linear optimal controllers for hybrid systems," in Proc. American Contr. Conf., (Chicago, IL), pp. 1190-1194, June 2000.

[11] T. A. Johansen, I. Petersen, and O. Slupphaug, "Explicit suboptimal linear quadratic regulation with state and input constraints," SINTEF report STF72-A00303, 1999.

[12] A. Bemporad and C. Filippi, "Suboptimal explicit MPC via approximate multiparametric quadratic programming," in Proc. 40th IEEE Conf. on Decision and Control, (Orlando, Florida), 2001. [13] F. Borrelli, M. Baotic, A. Bemporad, and M. Morari, "Efficient on-line computation of explicit model predictive control," in Proc. 40th IEEE Conf. on Decision and Control, (Orlando, Florida), 2001.

[14] M. Sznaier and M. J. Damborg, "Suboptimal control of linear systems with state and control inequality constraints.," Proc. 26th Conf. Decision and Control., pp. 761-762, 1987.

[15] D. Chmielewski and V. Manousiouthakis, "On constrained infinite-time linear quadratic optimal control.," Systems and Control Letters, vol. 29, pp. 121-129, 1996.

[16] P. Scokaert and J. B. Rawlings, "Constrained linear quadratic regulation.," IEEE Trans. Automatic Control', vol. 43, pp. 11631169,1998

[17] A. V. Fiacco, Introduction to sensitivity and stability analysis in nonlinear programming. Orlando, FL: Academic Press Inc., 1983.

[18] P. Tøndel, T. A. Johansen, and A. Bemporad, "An algorithm for multi-parametric quadratic programming and explicit MPC solutions," Tech. report 2001-7-W. Available at http://www.itk.ntnu.no/ansatte/Johansen_Tor.Arne/mpQPAlg.pdf, 2001. 